\title{
A comparison of learned aversions to gustatory and exteroceptive cues in rats
}

\author{
JANET D. LARSEN and THOMAS S. HYDE \\ Case Western Reserve University, Cleveland, Ohio 44106
}

\begin{abstract}
The experiment provides a direct comparison of the ability of subjects (rats) to associate gustatory and exteroceptive stimuli with illness. Previous experiments which have made similar comparisons between gustatory and exteroceptive cues have suffered from certain methodological problems involving stimulus control and compounding. The present experiment utilized a between-subjects design wherein half of the subjects had an auditory cue associated with poisoning and half had a taste cue. In both cases, the other cue was present, but was not predictive of poisoning. The auditory cue, like the taste cue, occurred only during drinking. This comparison was made in both an immediate and a delayed poisoning situation. The experiment found that while subjects were able to quickly associate a taste cue with illness, they were unable to form a similar association between poisoning and the exteroceptive stimulus. Results also showed that subjects will fail to acquire a taste aversion to a novel and salient gustatory cue when that cue is followed by illness only $50 \%$ of the time. This latter effect was more pronounced in the delayed poisoning situation.
\end{abstract}

There has been recent discussion of biologically determined differences in the ability of an organism to learn associations between different classes of events. Seligman (1970), for example, has argued for a dimension of "preparedness" in the acquisition of associative connections. Seligman, as a major case in point, refers to a body of literature often called "taste aversion learning," wherein a number of experiments have shown that subjects quickly learn an aversion to gustatory stimuli which are followed by illness, but do not learn a similar aversion to exteroceptive stimuli which accompany ingestion.

These conclusions are based on two types of studies. In the first type, typified by Garcia and Koelling (1967), subjects were poisoned after drinking water with a distinctive taste or after drinking water in the presence of constant environmental cues. Subjects readily learned to avoid the taste, but not to avoid drinking in the presence of the exteroceptive stimuli. In a second type of study, typified by Garcia and Koelling (1966), subjects were made ill after drinking water in the presence of a compound stimulus composed of gustatory, auditory, and visual components. In testing the elements of the compound, subjects typically avoided the taste, but not the ingestion of water in the presence of the exteroceptive stimuli. These types of results have been found in experimental situations where the poisoning occurred immediately after ingestion, and also in situations where the poisoning was delayed as long as $45 \mathrm{~min}$. Seligman (1970) and

Requests for reprints should be sent to Thomas S. Hyde, Department of Psychology NI-25, University of Washington, Seattle, Washington 98195. others have concluded that the acquisition of a tasteillness association is biologically predisposed, or "prepared," while that between exteroceptive stimuli and illness is "contraprepared" and difficult or impossible to establish.

While there seems to be a good deal of support for the above contention, both types of experiment have certain methodological problems and lend themselves to simpler explanations. The outcome of the first type of experiment (Garcia \& Koelling, 1967), where subjects learn to avoid substances with a distinctive taste, but not to avoid drinking in an environment where they have been poisoned, would be expected, given the evidence that subjects seldom learn continuous features of their environment as discriminative stimuli (see Jenkins \& Harrison, 1960). In the second procedure, where subjects are presented with a complex stimulus composed of gustatory and exteroceptive elemènts, problems associated with stimulus compounding must be considered. As Baker (1968) has pointed out, when any compound stimulus is used, the experiment has no control over which element(s) subjects will use as a cue. Kamin (1969) presents a cogent rationale for such an "overshadowing" phenomenon, and relates it to the blocking effects he has demonstrated in Pavlovian conditioning. This latter "taste aversion learning" procedure therefore shows only that when a complex stimulus composed of auditory, visual, and gustatory cues is followed by illness, it is the gustatory element that becomes the conditioned stimulus.

The taste aversion literature, therefore, has provided evidence that: (1) subjects readily acquire an aversion to taste stimuli which are followed by poisoning; (2) exteroceptive stimuli which are a 
constant feature of the environment do not become cues for poisoning; and (3) when gustatory and exteroceptive cues are presented as a compound, subjects will select the gustatory component as the salient cue.

There is, however, only limited evidence that subjects cannot readily learn to associate exteroceptive cues to illness when the exteroceptive event occurs discretely during drinking and when it is the only stimulus which provides information about poisoning. Domjan and Wilson (1972) have provided some evidence that the "taste aversion" phenomenon can be demonstrated in an experimental design free of these methodological problems. They used a between-subjects design, in which each subject experienced only one discrete stimulus (saccharin in water or a tone which sounded during water presentation) as a cue for poisoning. In an immediate poisoning situation, they found that the gustatory cue was more readily "associated" with poisoning than the auditory cue. Also, in their experiment, liquid was presented to all subjects directly, via a permanently implanted cannula. They measured the effects of poisoning in a preference testing situation following training.

The present experiment is an attempt to extend this finding and compare the relative "associability" of gustatory and exteroceptive cues to illness in an experimental situation free of methodological problems found in previous research, and quite different from the design used by Domjan and Wilson (1972). In the experiment, subjects were poisoned following ingestion of water where either a discrete auditory stimulus during drinking or a gustatory stimulus was predictive of poisoning. The comparison was made between subjects, one group having a gustatory stimulus predictive of poisoning and the other the auditory stimulus. Unlike Domjan and Wilson (1972), both the auditory and gustatory stimuli were presented to all subjects, but only one was predictive of poisoning. Also, rather than using a preference test for the effects of poisoning, the experiment measured the avoidance of drinking to the gustatory and auditory cues over the course of training. Further, these comparisons were made in both an immediate and a delayed poisoning condition.

\section{METHOD}

\section{Subjects and Apparatus}

The subjects were 24 male albino rats, 90 days old at the beginning of the experiment. The experiment was conducted in two identical $25 \times 25 \times 22 \mathrm{~cm}$ drinking boxes, with metal grid floors and Plexiglas covers. On one wall of each chamber was a houselight and a hole through which a drinking tube could be inserted. A calibrated water bottle was attached to the outside of this wall. On the opposite wall of each chamber was a small speaker. A drinkometer circuit was attached between the grid floor and the drinking tube.

\section{Procedure}

The experiment was performed in three complete replications of eight subjects each. Two subjects in each replication were assigned randomly to each of the four experimental conditions, one subject being assigned to each of the two drinking boxes.

Prior to the beginning of the experiment, the subjects were placed on water-deprivation schedules in the home cage for 7 days, during which they were allowed access to water for $20 \mathrm{~min}$ daily. The subjects were next habituated to the drinking boxes for 7 consecutive days. They were placed in the boxes for $10 \mathrm{~min}$ each day, and were allowed access to water. Two hours later, they were given an additional $20 \mathrm{~min}$ access to water in their home cages. During the last 4 days of habituation, all subjects were given an injection of sterile water under the same conditions as injections would be given in the experiment.

A 2 by 2 factorial experiment contained six subjects in each of the four conditions. One variable was the type of stimulus (taste or auditory) which would serve as a cue for poisoning, and the other was immediate or delayed poisoning. Subjects in all four treatment conditions were given 20 consecutive daily testing sessions in the drinking boxes, each $10 \mathrm{~min}$ in duration. On half of these sessions, the subjects were poisoned (sick days), and on the other half, they were not (safe days). Safe and sick days were randomized, with the restriction that the same condition not occur more than 3 consecutive days. Subjects were also counterbalanced with respect to safe and sick days, such that half of the subjects in each experimental condition received the opposite condition on each test day.

In Group 1 (taste relevant, immediate sickness), the taste of the solution served as a cue for poisoning. For half the subjects in this group, a salt taste served as the cue, while for the other half, it was a sweet taste. The subjects received the other (nonpoisoned) taste on safe days. The salt taste was made by mixing 7-g of sodium chloride per liter of distilled water, and the sweet taste by mixing 1-g of sodium saccharin per liter of water. These two tastes have been found to be equally preferred in rats (Garcia, Kovner, \& Green, 1970). Two minutes after the onset of the 10-min session, all subjects were briefly removed from the boxes and given an interperitoneal injection of apomorphine hydrochloride $(7.5 \mathrm{mg} / \mathrm{kg}$ of body weight) on sick days and an injection of sterile water on safe days. The apomorphine generally produced severe nausea within $60 \mathrm{sec}$ after the injection. For the subjects in this group, the auditory stimulus (20-pps clicker) was irrelevant to poisoning, and was presented whenever subjects made contact with the drinking tube on both sick and safe days.

In Group 2 (taste relevant, delayed sickness), the procedure was the same as for Group 1, except that the injection (apomorphine or sterile water) was given in the home cage 45 min after removal from the drinking box.

In Group 3 (sound relevant, immediate sickness), an auditory clicker $(20 \mathrm{pps})$ served as the cue for poisoning. The clicker sounder each time the subjects made contact with the drinking tube on days when the subject was to be poisoned, but not on safe days. Taste was made irrelevant by holding it constant across safe and sick days. Half of the subjects always received the salt taste; the other half, always received the sweet taste. As in the first condition, the subjects were given an injection 2 min after the beginning of the session, apomorphine on sick days and sterile water on safe days.

In Group 4 (sound relevant, delayed sickness), the procedure was exactly the same as in Group 3 , except that the injections were delayed for $\mathbf{4 5} \mathrm{min}$ after the end of the session in the drinking box.

The amount of water consumed in the drinking box was measured to the nearest milliliter for all subjects, both after the initial 2 min and after the entire 10-min session. All subjects were allowed $20 \mathrm{~min}$ access to water in their home cages approximately $2-h$ after their session in the drinking box. 

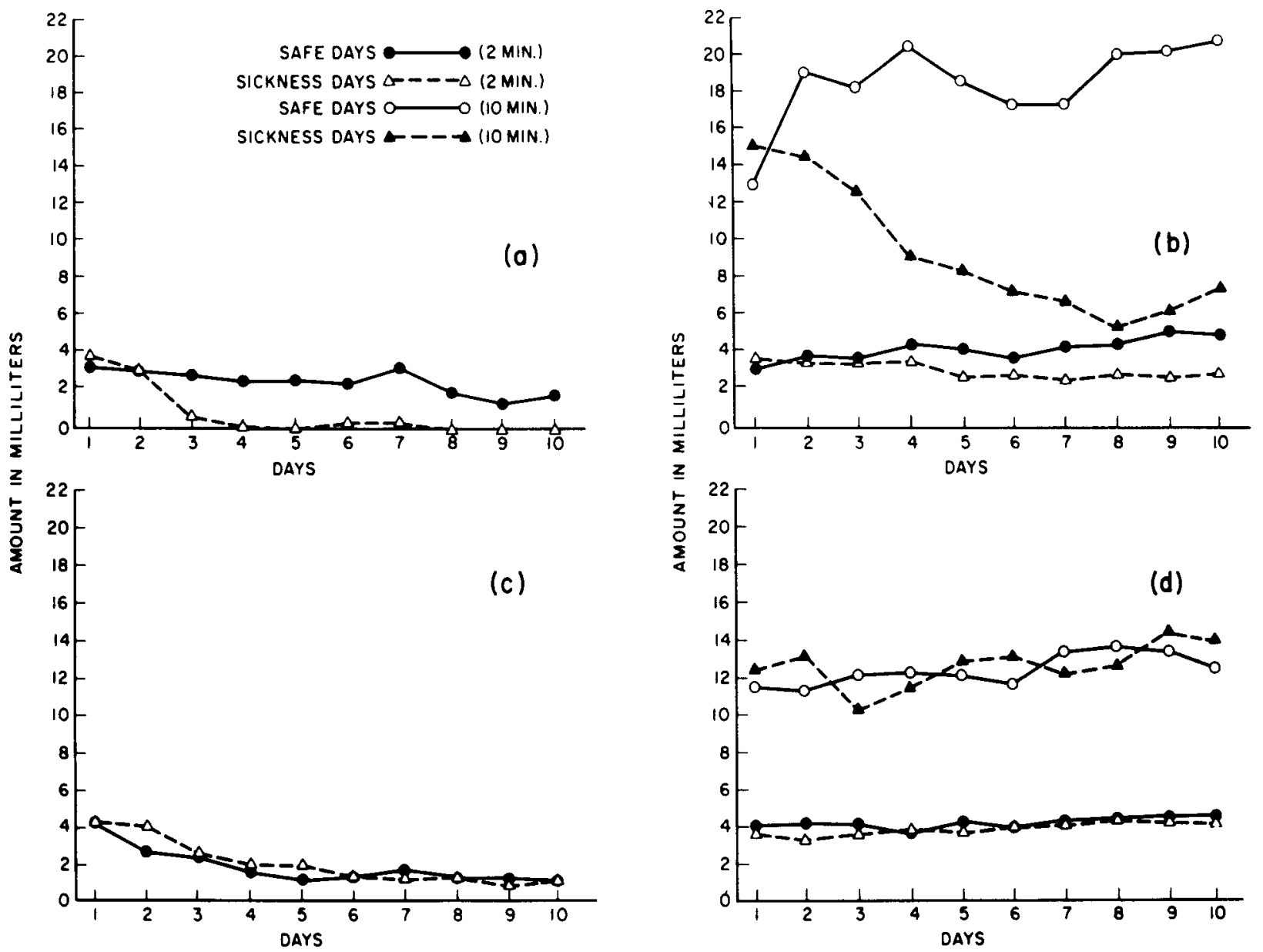

Figure 1. The average amount consumed, to the nearest milliliter, over the 10 safe and 10 sick days during the initial 2 min of each session for Group 1 (a), Group 2 (b), Group 3 (c), and Group 4 (d). The average amount consumed for the entire 10-min session is also shown for Group2 (b) and Group 4 (d).

\section{RESULTS}

Figure 1 presents the results for each of the four experimental conditions. For the two immediate sickness conditions (Groups 1 and 3 ) shown in panels $a$ and $c$, the figure plots the average amount consumed during the first 2 min (prior to the injection) on each of the 10 safe and 10 sick days. For the delayed sickness conditions (Groups 2 and 4) shown in panels $b$ and $d$, the figure plots the amount consumed during both the initial 2 min and during the entire 10-min session. The amount consumed during the entire session was not considered for the two immediate sickness groups because the subjects always stopped drinking immediately upon becoming ill.

Four separate three-way repeated measures ANOVAs (Replications by Cue by Sessions) were performed on the amount consumed during the initial 2 min for each of the experimental groups. Two additional ANOVAs were performed on the amount consumed during the entire session for the two delayed sickness conditions.
For Group 1 (taste relevant, immediate sickness), the subjects drank less during the cue for poisoning $[F(1,3)=152.58, p<.01]$, drank less over sessions $[F(3,27)=8.73, p<.01]$, and showed an interaction effect between cue and sessions $[F(3,27)=4.36$, $\mathrm{p}<.01$ ]. For Group 2 (taste relevant, delayed sickness), for the initial 2 min of the session, while subjects did not show a statistically significant effect for cue (safe vs sick) or for sessions, there was a statistically significant Cue by Sessions interaction effect $[F(3,27)=2.77, p<.05]$. Over the entire 10 -min session, the subjects drank less during the poison cue $[F(1,3)=98.32, p<.01]$, less over sessions $[\mathrm{F}(3,27)=2.93, \mathrm{p}<.01]$, and showed an interaction between cue and sessions $[F(3,27)=9.70$, $\mathrm{p}<.01$ ]. For Group 3 (sound relevant, immediate sickness), there was no difference in the amount subjects consumed to the safe and sick auditory cues, nor was there a significant interaction between session and cue. Subjects in this condition did consume less and less over sessions $[F(3,27)=9.46, p<.01]$. Finally, for Group 4 (sound relevant, delayed sickness), the analyses produced no statistically signifi- 
cant effects, for either the initial 2 min or the entire session.

\section{DISCUSSION}

The results for this experiment lend additional support to the contention that while subjects are "prepared" to acquire an association between gustatory stimuli and poisoning, they find it difficult, if not impossible, to acquire an association between exteroceptive stimuli and illness. In both immediate and delayed poisoning conditions wherein taste was the relevant cue to poisoning (Groups 1 and 2), the subjects quickly learned to stop drinking in the presence of the appropriate gustatory cue. However, when an auditory stimulus served as the cue to poisoning (Groups 3 and 4), no such association was learned.

In the present study, the auditory cue, like the taste cue, occurred only during drinking and was not compounded with a relevant taste cue. Yet subjects were still unable to acquire an association between exteroceptive stimuli and poisoning. This is not to say that such an association may not have been learned eventually. However, the present study demonstrated that after 20 daily training sessions, there was not even the slightest hint of an effect. These data provide a systematic replication of Domjan and Wilson (1972) using a different training procedure and a much different method of testing for the taste aversion effect.

Another interesting, and previously unreported, finding was that subjects did not develop a taste aversion in the presence of a salient and novel gustatory cue for poisoning when that cue was followed by illness only $50 \%$ of the time. This finding emerged in the two "sound-relevant" conditions (Groups 3 and 4), in which subjects experienced the same novel taste cue throughout the entire series of training sessions. Not only did the subjects continue to drink in the presence of the novel auditory stimulus, but they also kept drinking in the presence of a novel gustatory stimulus which was followed by poisoning $50 \%$ of the time. While there was some initial decline in the immediate poisoning condition (Group 3), the subjects consumed a constant amount over the last 12 test sessions. Moreover, there was no decline at all over sessions in the delayed poisoning condition (Group 4). This failure to acquire an aversion to a gustatory cue which is associated with poisoning only $50 \%$ of the time seems to add a new dimension to the taste aversion learning literature. To add to what Seligman (1970) has previously argued about the adaptiveness of a prepared connection between gestation and poisoning, such an "inconsistency" phenomenon would seem to add to the adaptiveness of taste aversion learning. If the taste aversion effect did not occur when there was some inconsistency in the relationship between the novel gustatory stimulus and illness, it would help to prevent spurious associations from developing. Given that the taste aversion effect is learned quickly and is difficult to extinguish, it would seem beneficial, particularly in delayed poisoning situations, that any inconsistency between poisoning and illness, particularly in the first few occurrences of the taste, would disrupt the effect. This finding would seem to be deserving of further exploration.

\section{REFERENCES}

Baker, T. W. Properties of compound conditioned stimuli and their components. Psychological Bulletin, 1968, 70, 611-625.

Domjan, M., \& Wilson, N. E. Specificity of cue to consequence in aversion learning in the rat. Psychonomic Science, 1972, 26, 143-145.

Garcia, J., \& Koelling, R. A. Relation of cue to consequence in avoidance learning. Psychonomic Science, 1966, 4, 123-124.

GaRcia, J., \& Koelling, R. A. A comparison of aversions induced by X-rays, toxins, and drugs in the rat. Radiation Research Supplement, 1967, 7, 439-450.

Garcia, J., Kovner, R.. \& Green, K. F. Cue properties vs. palatability of flavors in avoidance learning. Psychonomic Science, 1970, 20, 313-314.

Jenkins, H. M.. \& HaRRISon. R. H. Effect of discrimination training on auditory generalization. Joumal of Experimental Psychology, 1960, 59, 246-253.

Kamin, L. J. Predictability, surprise, attention and conditioning. In Byron A. Campbell \& Russell M. Church (Eds.), Punishment and aversive behavior. New York: AppletonCentury-Crofts, 1969.

Seligman, M. E. P. On the generality of the laws of learning. Psychological Review, 1970, 77, 406.418.

(Received for publication March 29. 1976; revision received August 1, 1976.) 